\title{
Estimating Lighting Direction and Classifying Textures
}

\author{
Mike Chantler ${ }^{1}$, Ged McGunnigle ${ }^{1}$, Andreas Penirschke ${ }^{2}$ and Maria Petrou ${ }^{3}$ \\ ${ }^{1}$ Texture Lab., Heriot-Watt University, Edinburgh, Scotland \\ http: //www.cee.hw.ac.uk/texturelab/ \\ ${ }^{2}$ Darmstadt University of Technology, Germany \\ ${ }^{3}$ Department of Electronic and Electrical Engineering, University of Surrey \\ M.Petrou@ee.surrey.ac.uk
}

\begin{abstract}
The appearance of a rough surface is affected by the direction from which it is lit and texture classifiers should account for this. We propose a classifier that is robust to lighting direction-even when the direction is unknown. An existing model of the dependency of texture features on lighting direction is used to develop a probabilistic model. Given a feature set, the algorithm estimates the most likely illumination direction for each texture class. The likelihoods of each candidate (with their estimated lighting) are compared to classify the sample. The ability of the classifier to identify illuminant direction, and to assign the correct class, was tested on 25 real texture samples. The classifier was able to accurately estimate both the azimuth and the zenith of the light source for most textures and gave a $98 \%$ classification rate.
\end{abstract}

\section{Introduction}

This paper deals with the classification of rough surface textures on the basis of their image texture. Although many texture techniques have been applied implicitly to this type of texture - the majority of Brodatz textures [1] contain at least a component due to surface topography-little work has been carried out on the phenomena associated with this group. One characteristic of rough surface textures is that the appearance of the surface is a function of the illuminant direction as well as of the surface topography, Figure 1, [2] [3]. If the image is affected by the direction of lighting, then features drawn from the image will also be affected. The same surface may be classified as belonging to different classes depending on the direction from which it was lit. The effect can be modelled and either accounted for [4], or counteracted [5], if the direction of the illumination is known. However, in many cases this information is not available and the aim of this paper is to develop a classifier that can classify rough surface textures consistently, without needing to know from where they are lit.

Little work has been published on this subject. Dana, Nayer, van Ginneken and Koenderink established the Columbia-Utrecht database of real world surface textures which they used to investigate bidirectional texture functions [6]. Later they developed histogram [7, 8] and correlation models [3] of these textures. Leung and Malik developed a 

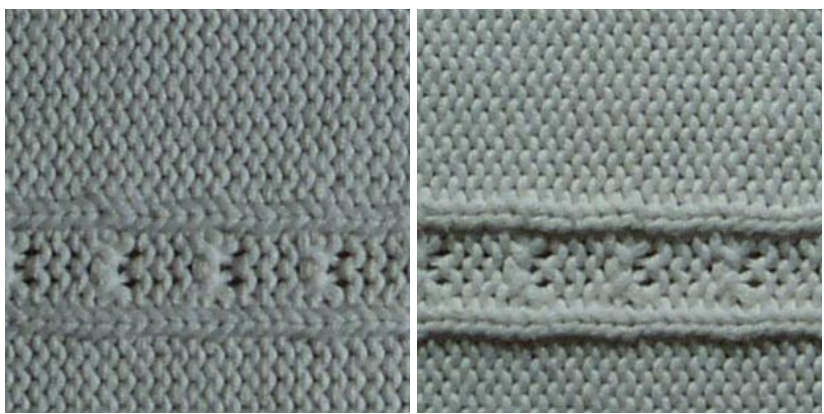

Figure 1: Images of a sample lit from different azimuths (or tilt angles).

texture classification scheme that identifies 3D 'textons' in the Columbia-Utrecht database for the purposes of illumination and viewpoint invariant classification [9, 10]. Chantler et al. modelled the effect of azimuth on features [11]. Penirschke et al. used this model to develop a classifier that is able to classify surfaces that are lit by light sources whose azimuth is unknown, [12].

This paper proposes a technique that is robust to the lighting direction-even when the direction is not known. An existing, deterministic, model of the effect of the light source direction on texture features is expressed in probabilistic terms. That is, for a given texture, under known lighting conditions, we can state the probability of a feature value, and by extension, the probability of a particular feature vector. Using Bayes' theorem, given a feature vector, we can therefore find the most likely lighting direction for each class of texture. To classify, we assume that the test sample belongs to each texture class in turn and estimate the most likely lighting direction given that assumption. By comparing the relative likelihoods of each candidate (and their associated optimal lighting direction) we can estimate to which class the test sample belongs, and implicitly from which direction it was lit.

We use 25 real textures to assess how well the classifier can identify the source direction and classify the sample. The algorithm was found to be effective for azimuth estimation, zenith estimation was poorer; though the data set was limited. The classifier was applied to the samples under 24 different lighting directions and achieved a classification rate of $98 \%$.

This paper presents and evaluates a technique for classifying rough surface textures regardless of the direction from which they are lit. The classification was found to be robust to illuminant azimuth and, within the experimental range, robust to the zenith of the light source. The technique does not require the lighting direction to be known a priori, however, it does require more training and computation than a conventional classifier.

\section{Modelling the Feature Vector}

Consider a classification task where a sample is imaged from directly overhead and lit by a source with azimuth (or tilt) $\tau$ and zenith (or slant) $\sigma$, Figure 2. The classifier uses a feature vector composed of $i$ features. Each feature is the estimated variance of an image produced by convolving the input image with one of a set of linear filters $h_{i}$. 

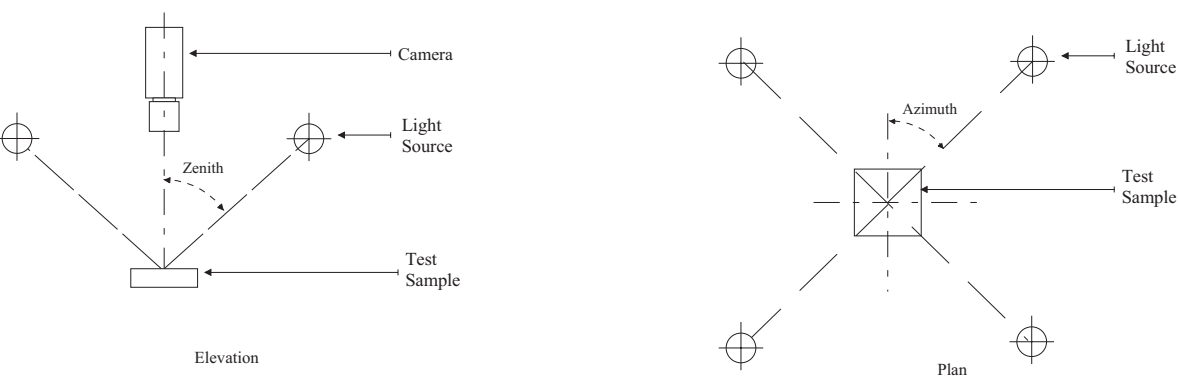

Figure 2: Image capture setup.

It has been shown that this type of feature is a function of the tilt and slant of the light source [13]. The dependency on tilt was modelled in [11], the dependency on slant and tilt is approximated by Equation 1, [14].

$$
f(\tau, \sigma)=a \sin ^{2} \sigma+b \cos 2 \tau \sin ^{2} \sigma+c \sin 2 \tau \sin ^{2} \sigma
$$

where the parameters $a, b$ and $c$ are functions of the surface height function and the linear filter of the texture feature.

If the tilt of the light source is varied, the feature vector will trace out a hyperellipse in $i$-dimensional feature space [11]. Figure 3 shows the behaviour of two Gabor filters (with the same centre frequency, but oriented at $0^{\circ}$ and $45^{\circ}$ ) as a function of illuminant tilt for six real textures. It shows the elliptical behaviour of the cluster means. Clearly, this variation will cause a linear classifier to fail.

In practice the feature will differ from the model's prediction. We model the difference as a zero mean, normally distributed random variable with standard deviation $s$. We can now express the relationship between the feature and lighting direction for a given texture class $k$ in probabilistic terms, Equation 2.

$$
p_{k}\left(f_{i} \mid \tau, \sigma\right)=\frac{1}{s \sqrt{2 \pi}} \exp \left[-\frac{\left[f_{i}-\sin ^{2} \sigma\left(a_{i}+b_{i} \cos 2 \tau+c_{i} \sin 2 \tau\right)\right]^{2}}{2 s^{2}}\right]
$$

where $p_{k}\left(f_{i} \mid \tau, \sigma\right)$ is the probability of the event of feature $i$ having value $f_{i}$ occuring, given that the texture $k$ is lit from $(\tau, \sigma)$.

The feature vector, $F$, is composed of $i$ features. Assuming these are orthogonal the joint distribution can be described using Equation 3

$$
p_{k}(F \mid \tau, \sigma)=\prod_{i} \frac{1}{s \sqrt{2 \pi}} \exp \left[-\frac{\left[y_{i}-\sin ^{2} \sigma\left(a_{i}+b_{i} \cos 2 \tau+c_{i} \sin 2 \tau\right)\right]^{2}}{2 s^{2}}\right]
$$

\section{Classification}

The classifier is trained by parameterising the model for each candidate class. Each texture sample must be imaged under different illumination directions and features calculated 


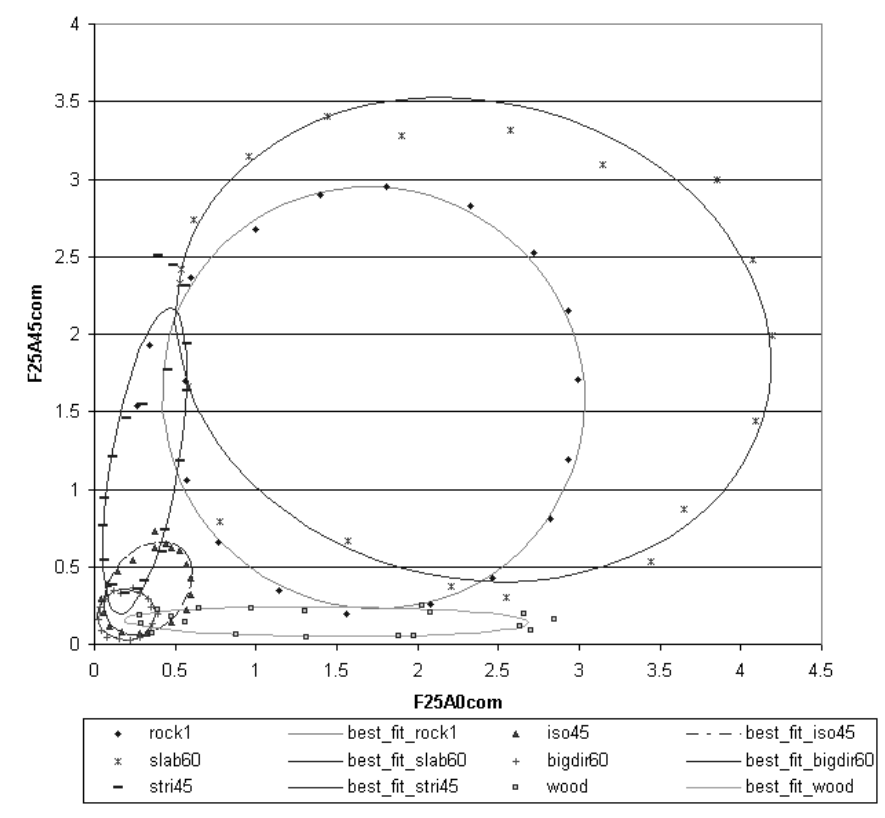

Figure 3: The behaviour of six textures in the comF25A0/comF25A45 feature space together with the best fit ellipses (each point on an ellipse denotes a different value of illuminant tilt)

from these images. We recommend that at least three images should be taken at two or more slants. In this work we use 12 images at two slant angles. The parameter values of the model are calculated to give the best fit to the data. This allows us to predict the likelihood of a particular feature value, for a given texture class, lit from a given direction.

Presented with a feature vector, the classifier uses a probabilistic model to identify the most likely lighting direction and texture class. The probability of a lighting direction, given a particular feature vector can be related to Equation 3 using Bayes' theorem, Equation 4.

$$
P_{k}(\tau, \sigma \mid F)=\frac{P_{k}(F \mid \tau, \sigma) P_{k}(\tau, \sigma)}{P_{k}(F)}
$$

Now, assuming all lighting directions are, a prior, equally likely, $P(\tau, \sigma)$ is constant. And because, we are only interested in the relative probabilities of the values of $\sigma$ and $\tau$ at a given $F$ we may replace $P_{k}(F)$ with a constant, i.e.

$$
P_{k}(\tau, \sigma \mid F)=\alpha P_{k}(F \mid \tau, \sigma)
$$

The most likely direction of the light source, $\hat{\tau}, \hat{\sigma}$ for each texture is estimated by maximising the likelihood function of that texture.

$$
\hat{\tau}, \hat{\sigma}=\underset{\tau, \sigma}{\operatorname{ArgMax}} P_{k}(F \mid \tau, \sigma)
$$


The numerical optimisation is simplified by instead maximising the $\log$ likelihood function, Equation 3.

$$
\ln p_{k}(F \mid \tau, \sigma)=\ln \prod_{i}\left(\frac{1}{s_{i} \sqrt{2 \pi}}\right)+\sum_{i} \frac{\left[f_{i}-\sin ^{2} \sigma\left(a_{i}-b_{i} \cos (2 \tau)-c_{i} \sin 2 \tau\right)\right]^{2}}{2 s_{i}^{2}}
$$

In addition, a trigonometric substitution is performed to transform the equation into a $12^{\text {th }}$ order polynomial. This is optimised using a standard Matlab routine.

We now have a series of $k$ competing hypotheses about the class of the sample and the direction it was lit from. Again, we are interested only in relative probabilities. If we assume the classes are, initially, equally likely, the most likely class can be identified by evaluating Equation 7.

$$
\hat{k}=\underset{k}{\operatorname{ArgMax}} P_{k}\left(F \mid \hat{\tau_{k}}, \hat{\sigma_{k}}\right)
$$

\section{Experiments}

The proposed classifier is assessed on two criteria: the accuracy with which it estimates the direction of the light source; and the accuracy with which it can assign a sample to the correct class. The experiments were carried out on 25 samples, shown at the end of this paper, imaged at slant angles of $45^{\circ}$ and $60^{\circ}$ and tilt angles of $30^{\circ}$ increments.

The classifier's features are estimates of the variance of images produced by filtering the input image with a set of Gabor filters. Gabor filters are Gaussians modulated by complex exponentials - they have a centre frequency $\omega$ and orientation $\phi$. In our nomenclature they are denoted by $\operatorname{com} F \omega A \phi$, where $\omega$ is specified in cycles per image and $\phi$ is

\begin{tabular}{|c|c|c|c|c|c|c|c|}
\hline \multirow{2}{*}{ filter } & \multicolumn{7}{|c|}{ Gabor filter bank } \\
\hline & 12 & 10 & 8 & 6 & 4 & 3 & 2 \\
\hline " comF20A0 & $\overline{\mathrm{XX}}$ & $\overline{\mathrm{X}}$ & $\overline{\bar{X}}$ & $\overline{\mathrm{X}}$ & $\overline{\mathrm{X}}$ & $\overline{\mathrm{X}}$ & $\overline{\bar{X}}$ \\
\hline comF20A45 & $\mathrm{X}$ & $X$ & $\mathrm{X}$ & & & & \\
\hline comF20A90 & $X$ & $X$ & $\mathrm{X}$ & $\mathrm{X}$ & $X$ & $X$ & $\mathrm{X}$ \\
\hline comF20A135 & $\mathrm{X}$ & $X$ & $\mathrm{X}$ & & & & \\
\hline comF30A0 & $\mathrm{X}$ & & & & & & \\
\hline comF30A45 & $\mathrm{X}$ & $\mathrm{X}$ & $\mathrm{X}$ & $\mathrm{X}$ & $X$ & $X$ & \\
\hline comF30A90 & $X$ & & & & & & \\
\hline comF30A135 & $\mathrm{X}$ & $\mathrm{X}$ & $\mathrm{X}$ & $\mathrm{X}$ & X & & \\
\hline comF40A0 & $\mathrm{X}$ & $\mathrm{X}$ & $\mathrm{X}$ & $\mathrm{X}$ & & & \\
\hline comF40A45 & $X$ & $X$ & & & & & \\
\hline comF40A90 & $X$ & $X$ & $\mathrm{X}$ & $\mathrm{X}$ & & & \\
\hline comF40A135 & $X$ & $\mathrm{X}$ & & & & & \\
\hline
\end{tabular}
in degrees. Sets of filters are combined into banks, Table 1.

Table 1: Gabor filter banks used for classification.

The accuracy of tilt estimation is shown in Figure 4 (top). $76 \%$ of the estimates were within $5^{\circ}$ of the correct value, $82 \%$ were within $10^{\circ}$ and only one texture sample was more 
than $20^{\circ}$ in error. The accuracy of slant estimation is shown in Figure 4 (bottom). There are several points to note regarding this. First, two training slants, separated by $15^{\circ}$ were used, however $26 \%$ of the tests were more than $7.5^{\circ}$ in error; secondly, estimation from $45^{\circ}$ was significantly more accurate than estimation from $60^{\circ}(52 \%$ of samples have less than $2^{\circ}$ of error for the $45^{\circ}$ case, compared to only $4 \%$ for the $60^{\circ}$ case); thirdly the image samples that perform poorly for tilt estimation correspond well to those that perform badly for slant estimation - these tend to be drawn from the $\mathrm{AD}^{*}$ and $\mathrm{AF}^{*}$ groups (repeating primitives and fabrics) both of which experience significant shadowing. The last two points suggest that the prime source of inaccuracy is shadowing.

The second, more important criterion for the classifier is classification accuracy. We applied 6 feature sets composed of between 3 and 12 Gabor filters to the data set, i.e. 25 samples lit from 24 different directions. The overall error rate is shown in Figure 5. The most effective feature vector, composed of 10 features, gave a $98 \%$ classification rate. Increasing the number of features gave a small increase in the error rate, but also led to numerical instability in the optimisation procedure. Reducing the number of features increased the error rate- with the most significant increase occurring for sets of less than 6 features.

\section{Conclusions}

We proposed a technique to classify rough surface textures that are lit from an unknown direction. The technique estimates the most likely illumination vector by optimising a probabilistic model for each class and classifies by comparing the optima of each class. The technique was effective in estimating the azimuth of the light source. Estimation of the zenith angle was less effective, though both the training and test data were much more limited. The evidence suggests that shadowing degrades the effectiveness of the system when the surface is lit from shallow angles. Nonetheless, the classifier is robust to changes in the lighting direction and is able to maintain a high level of accuracy. The most effective feature set consisted of 10 features (98\%): below 6 features was found to be insufficient, and vectors with more than 12 were prone both to a small increase in the error rate and to failures in the optimisation procedure. 

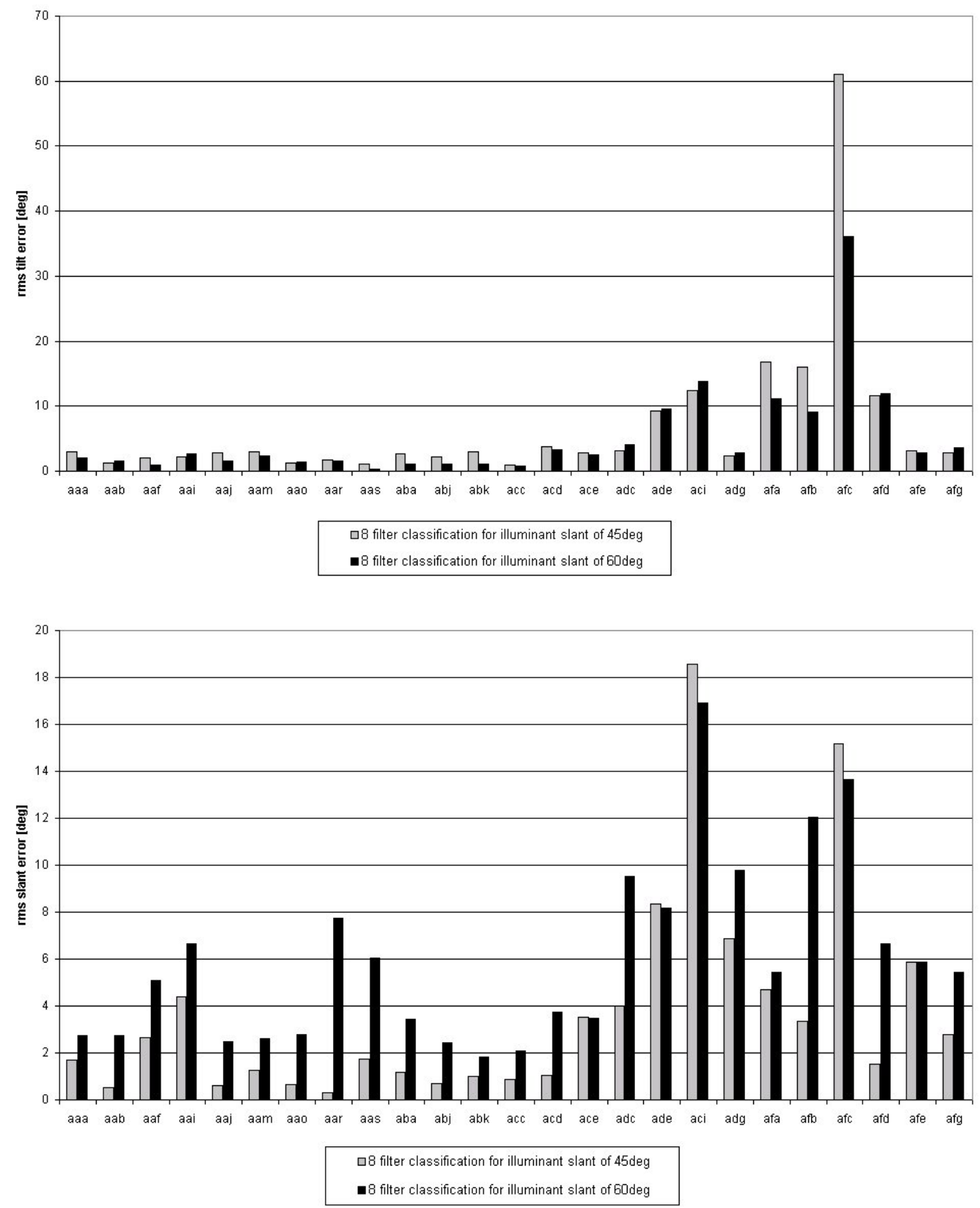

Figure 4: Root mean square, rms, tilt error (top) and rms slant error (bottom) for the tested surfaces 


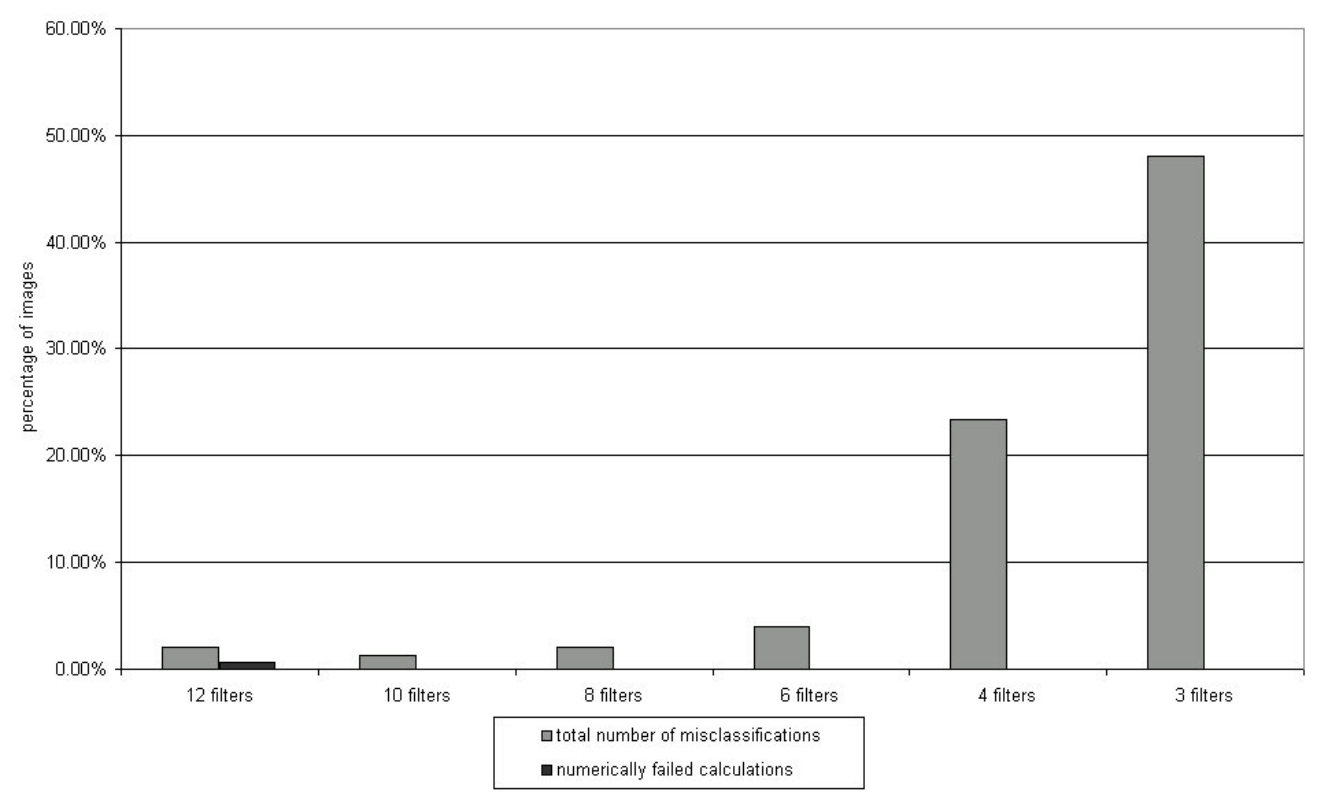

Figure 5: Percentage of misclassified images for illumination direction independent classification dependent on the used Gabor filter bank

\section{References}

[1] P. Brodatz. Textures: a photographic album for artists and designers. Dover, New York, 1966.

[2] M.J. Chantler. Why illuminant direction is fundamental to texture analysis. IEE Proc. Vision, Image and Signal Processing, 142(4):199-206, August 1995.

[3] K.J. Dana and S.K. Nayar. Correlation model for 3d texture. In Proceedings of ICCV99: IEEE International Conference on Computer Vision, pages 1061-1067, 1999.

[4] G. McGunnigle and M.J. Chantler. A model-based technique for the classification of textured surfaces with illuminant direction invariance. In Proceedings of BMVC97: British Machine Vision Conference, volume 2, pages 470-479, 1997.

[5] M.J. Chantler and G. McGunnigle. Compensation of illuminant tilt variation for texture classification. In Proceedings of 5th International Conference on image processing and its applications, pages 767-761, 1995.

[6] K.J. Dana, S.K. Nayar, B. van Ginneken, and J.J. Koenderink. Reflectance and texture of real-world surfaces. In Proceedings of IEEE Conference on Computer Vision and Pattern Recognition, pages 151-157, 1997.

[7] K.J. Dana and S.K. Nayar. Histogram model for 3d textures. In Proceedings of IEEE Conference on Computer Vision and Pattern Recognition, pages 618-624, 1998. 


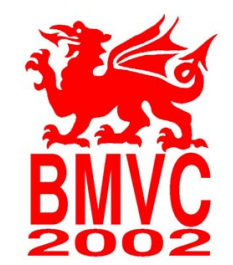

[8] B. van Ginneken, J.J. Koenderink, and K.J. Dana. Texture histograms as a function of irradiation and viewing direction. International Journal of Computer Vision, 31(2/3):169-184, April 1999.

[9] T. Leung and J. Malik. Recognizing surfaces using three-dimensional textons. In Proceedings of ICCV99: IEEE International Conference on Computer Vision, pages 1010-1017, 1999.

[10] T. Leung and J. Malik. Representing and recognizing the visual appearance of materials using three-dimensional textons. International Journal of Computer Vision, 43(1):29-44, June 2001.

[11] M.J. Chantler, M. Schmidt, M. Petrou, and G. McGunnigle. The effect of illuminant rotation on texture filters: Lissajous's ellipses. In ECCV2002, European Conference on Computer Vision, volume III, pages 289-303, 2002.

[12] A. Penirschke, M.J. Chantler, and M. Petrou. Illuminant rotation invariant classification of 3d surface textures using lissajous's ellipses. In TEXTURE 2002, The 2nd International Workshop on Texture Analysis and Synthesis, pages 103-107, 2002.

[13] M.J. Chantler. The effect of variation in illuminant direction on texture classification. Ph.D. thesis, Department of Computing and Electrical Engineering Heriot Watt University, 1994.

[14] A. Penirschke. Illumination invariant classification of 3d surface textures. Research Memorandum, (RM/02/4), 2002.

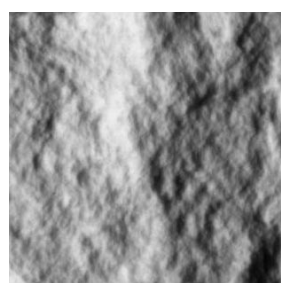

aaa

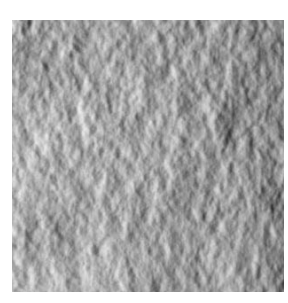

aаj

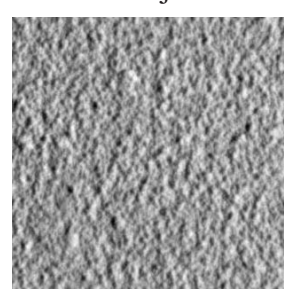

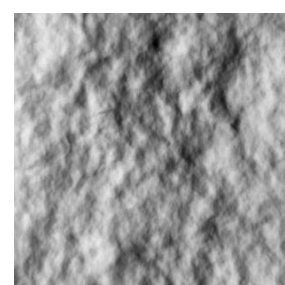

$\mathrm{aab}$

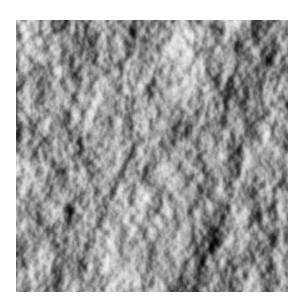

aam

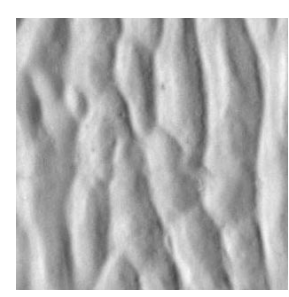

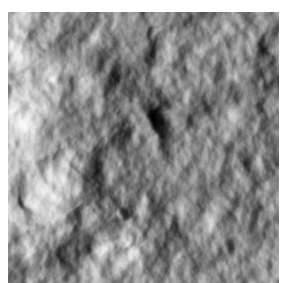

aaf

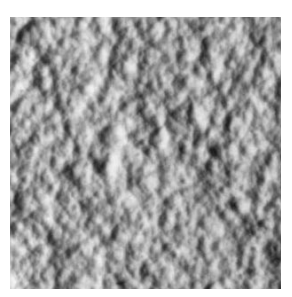

aao

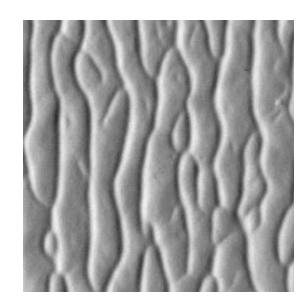

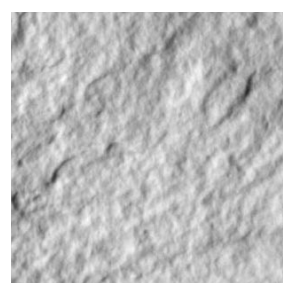

aai

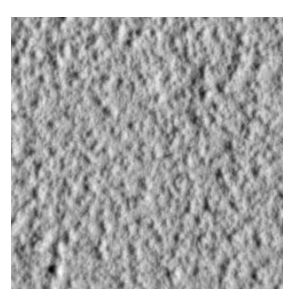

aar

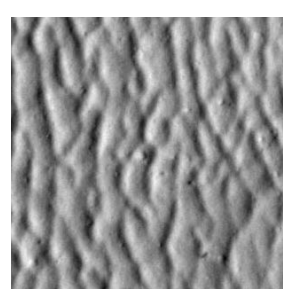



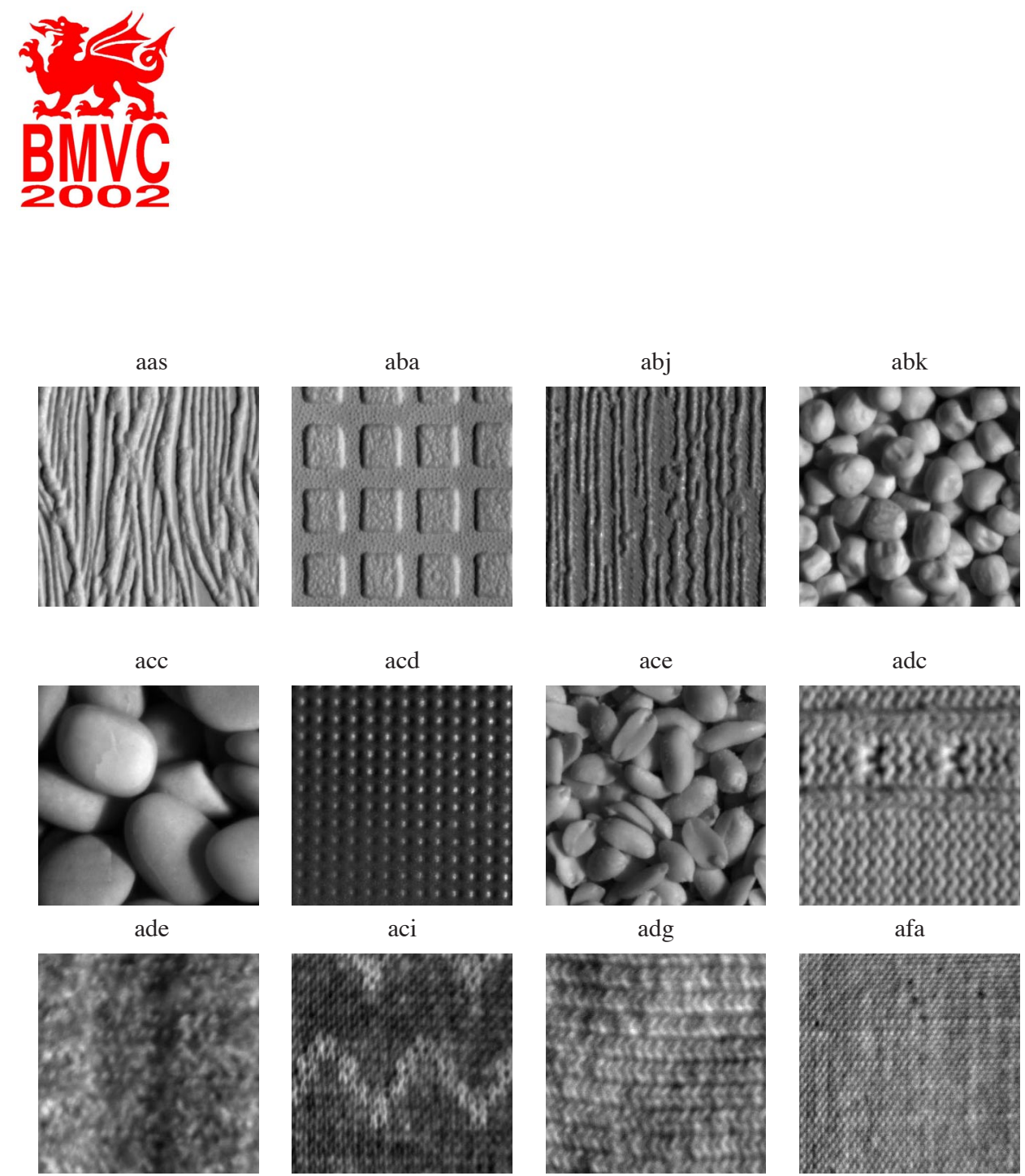

afa

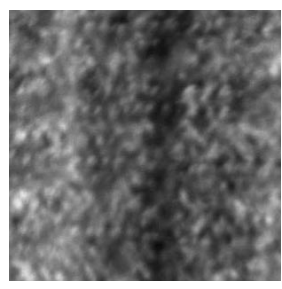

afc

afd

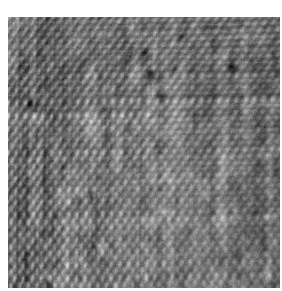

afe

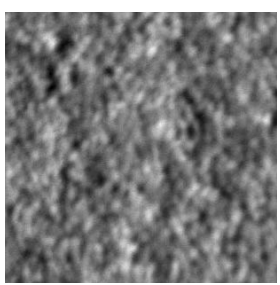

afg 